\title{
Precisão da retinoscopia sem lentes neutralizadoras na hipermetropia experimental de olho artificial
}

\author{
Accuracy limits of retinoscopy without neutralising lenses for \\ evaluation of experimental hypermetropia in a model eye
}

Beatriz Simões-Corrêa ${ }^{1}$, Augusto Duarte ${ }^{2}$,Alessandra Torres 3 , Alexandre Alvarez Escaneo ${ }^{3}$, Lilia Lucena ${ }^{3}$, Leonardo Ferreira da Costa $^{3}$, Marcio José Salgueiro ${ }^{3}$, Monick Goecking ${ }^{1}$

\section{RESUMO}

Objetivo: Avaliar a acurácia e a precisão da retinoscopia sem lentes neutralizadoras na hipermetropia experimental de olho artificial. Métodos: Observação da projeção da faixa do retinoscópio em olho artificial preparado para apresentar hipermetropias de $+1,00 \mathrm{D}$ a +8,00 D. A movimentação da faixa é feita simultaneamente com a variação da sua largura, deslocando a luva do retinoscópio até a obtenção da neutralização do movimento. O retinoscópio foi preparado para dois modos de medida: com escala milimétrica sob a luva e com acoplamento a potenciômetro ligado a multímetro de leitura digital em ohms (Ù). Oito médicos oftalmologistas realizaram 1200 retinoscopias em cada modo de medida, num total de 2400 . Calculou-se a média e o desvio padrão das observações. Para comparar os resultados em mm e em ohms (Ù) calculou-se o coeficiente de variação (CV) a partir dos desvios padrão das observações em cada modo. Resultados: Pelos modos descritos foi possível estimar hipermetropias até $+4,00$ dioptrias. Conclusão: A acurácia e a reprodutibilidade experimentais foram boas na identificação de hipermetropias até $+4,00 \mathrm{D}$.

Descritores: Retinoscopia/métodos; Ambliopia; Hiperopia

\begin{abstract}
Objective: Evaluate accuracy and reproducibility of streak retinoscopy without neutralising lenses in experimental hyperopia of an artificial eye. Methods: Observe a Welsh Allynretinoscope streak through the pupil of an artificial eye prepared do show hyperopia from $+1,00 \mathrm{D}$ to $+8,00 \mathrm{D}$. The streak was moved while varying its width by sliding the sleeve upwards until neutralisation point. The retinoscope was fitted either with a millimetre scale under the sleeve or with a multimetre connected to a rheostat driven by a spool snugly fastened around the sleeve. Multimetre readings were taken in ohms (Uे). Eight ophthalmologists performed 1200 in each mode totalysing 2400 readings. Average and standard deviation of the readings in each mode were determined, as well as the variation coefficient in order to allow comparison of results in millimetre and U.Results: Based on the proposed method it was possible to estimate hyperopia up to $+4,00$ D. Conclusion: Accuracy and reproducibility in experimental hyperopia of an artificial eye was feasible up to +4,00 D.

Keywords: Retinoscopy/methods; Amblyopia; Hyperopia
\end{abstract}

\footnotetext{
${ }^{1}$ Hospital Federal dos Servidores do Estado, Rio de Janeiro (HFSE) - Rio de Janeiro (RJ), Brasil;

${ }^{2}$ Pontifícia Universidade Católica do Rio de Janeiro (PUC) - Rio de Janeiro (RJ), Brasil;

${ }^{3}$ Programa de residência, Setor de Estrabismo do Hospital Federal dos Servidores do Estado (HFSE) - Rio de Janeiro (RJ), Brasil.

Trabalho realizado no Serviço de Oftalmologia do Hospital Federal dos Servidores do Estado - Rio de Janeiro (RJ), Brasil
}

Os autores declaram inexistir conflitos de interesse

Recebido para publicação em 28/7/2011 - Aceito para publicação em 30/5/2012 


\section{INTRODUÇÃO}

A retinoscopia sem lentes neutralizadoras é interes sante para a refratometria objetiva na criança que não coopera no exame ao refrator computadorizado ou que não permite a anteposição de lentes de caixa de provas ou de régua de Parenta em seus olhos durante a realização da retinoscopia convencional.

É possível realizá-la segundo o método descrito por Corboy $^{(1)}$, que estima a potência dióptrica neutralizadora pela largura aparente da faixa projetada no olho examinado. Wallace et al. ${ }^{(2)}$ relataram correlação entre os resultados clínicos obtidos pela retinoscopia com lentes nas ametropias entre $-3,12$ e $+6,00$ dioptrias (D), forte correlação até a hipermetropia de $+2,00 \mathrm{D}$ e seus equivalentes esféricos em estudo de retinoscopia sem lentes em 100 crianças examinadas, empregando retinoscópio de Copeland provido de escala numérica indicativa das potências dióptricas das lentes de prova usadas para calibração do deslocamento da luva ao exame de olho artificial.

O objetivo deste trabalho foi avaliar a acurácia e a precisão da retinoscopia sem lentes neutralizadoras na hipermetropia experimental de olho artificial.

\section{Métodos}

As retinoscopias foram realizadas com retinoscópiosWelch Allyn como se descreve a seguir.
Cada retinoscópio foi preparado sob duas formas:

1- Fixou-se sob a luva uma escala milimétrica vertical (Figuras 1 e 2). O ponto zero correspondia ao nível mais baixo da luva;

2- Encaixou-se sobre a luva um carretel que acionava o potenciômetro ligado ao multímetro (Figura 3). O deslocamento vertical da luva era apresentado numericamente em ohms (unidade de resistência elétrica, símbolo ) no mostrador digital do multímetro, correspondendo o ponto zero ao nível mais baixo da luva.

Cada olho artificial (Figura 4) consistia em dois cartuchos em forma de prisma retangular, encaixados telescopicamente, podendo deslizar um por dentro do outro. Tinha potência de $18 \mathrm{D}$ e pupila de $3 \mathrm{~mm}$. À frente da pupila um aro podia receber lentes da caixa de provas (diâmetro $38 \mathrm{~mm}$ ). Foram preparados a partir de modelo com potência dióptrica fixa construído pelo dr. Celso Cunha(Comunicação pessoal, 1996).

Colocou-se uma lente esférica $+2,00 \mathrm{D}$ da caixa de provas à frente da pupila do olho artificial que foi fixado sobre uma régua a $0,5 \mathrm{~m}$ do olho do médico examinador (Figura 5). Com a luva do retinoscópio no ponto mais baixo projetava-se a faixa através da pupila do olho artificial e movimentava-se o retinoscópio observando o movimento correspondente da faixa no fundo do olho artificial. Regulava-se então seu comprimento até obter neutralização do movimento. Retirava-se a lente $+2,00$ D; o olho artificial preparado desta maneira correspondia a olho emétrope.

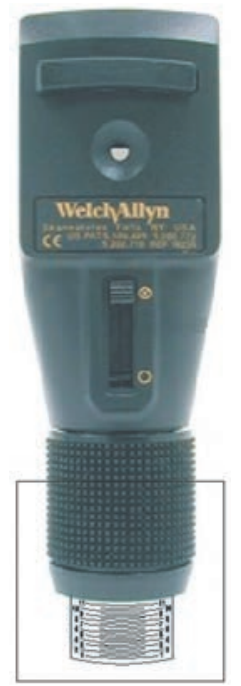

Figura 1: Escala milimétrica fixa ao retinoscópio

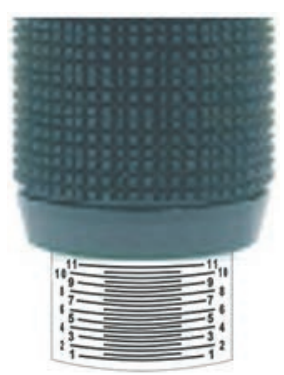

Figura 2:

- Pormenor

da área no retângulo da figura 1

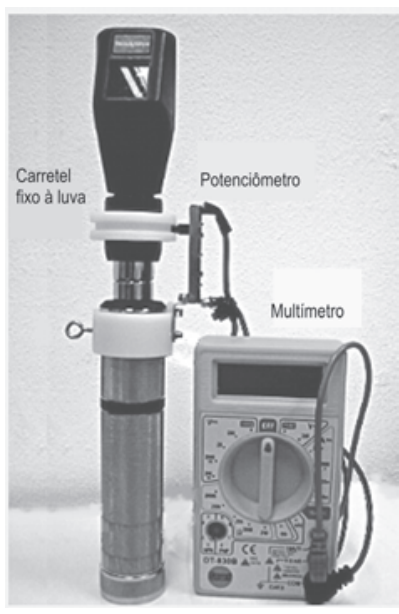

Figura 3: Retinoscópio montado
Figura 4: Olho artificial com lente $-3,00 \mathrm{D}$ encaixada no aro
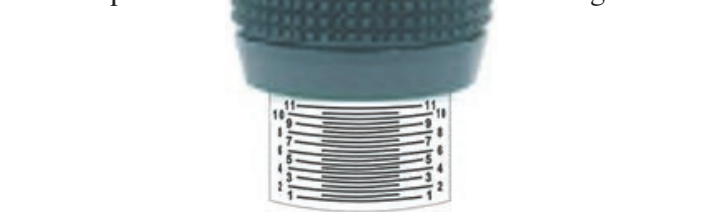

Figura 5:

Prática da retinoscopia sem lentes neutralizadoras

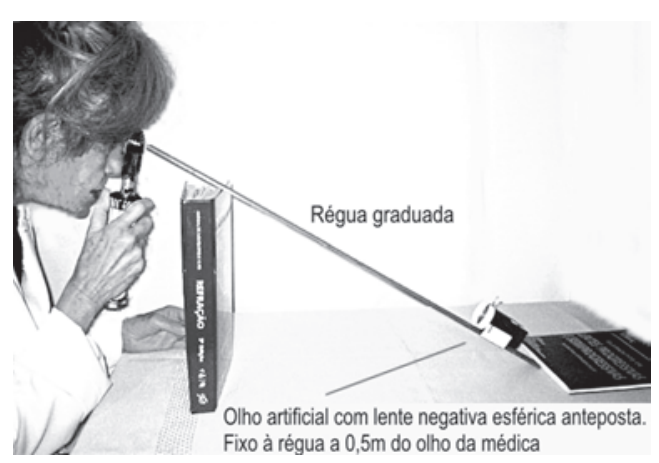


Tabela 1

Resultados da retinoscopia sem lentes com leitura em milímetros na escala do retinoscópio

Resultado das 20 leituras (mm)

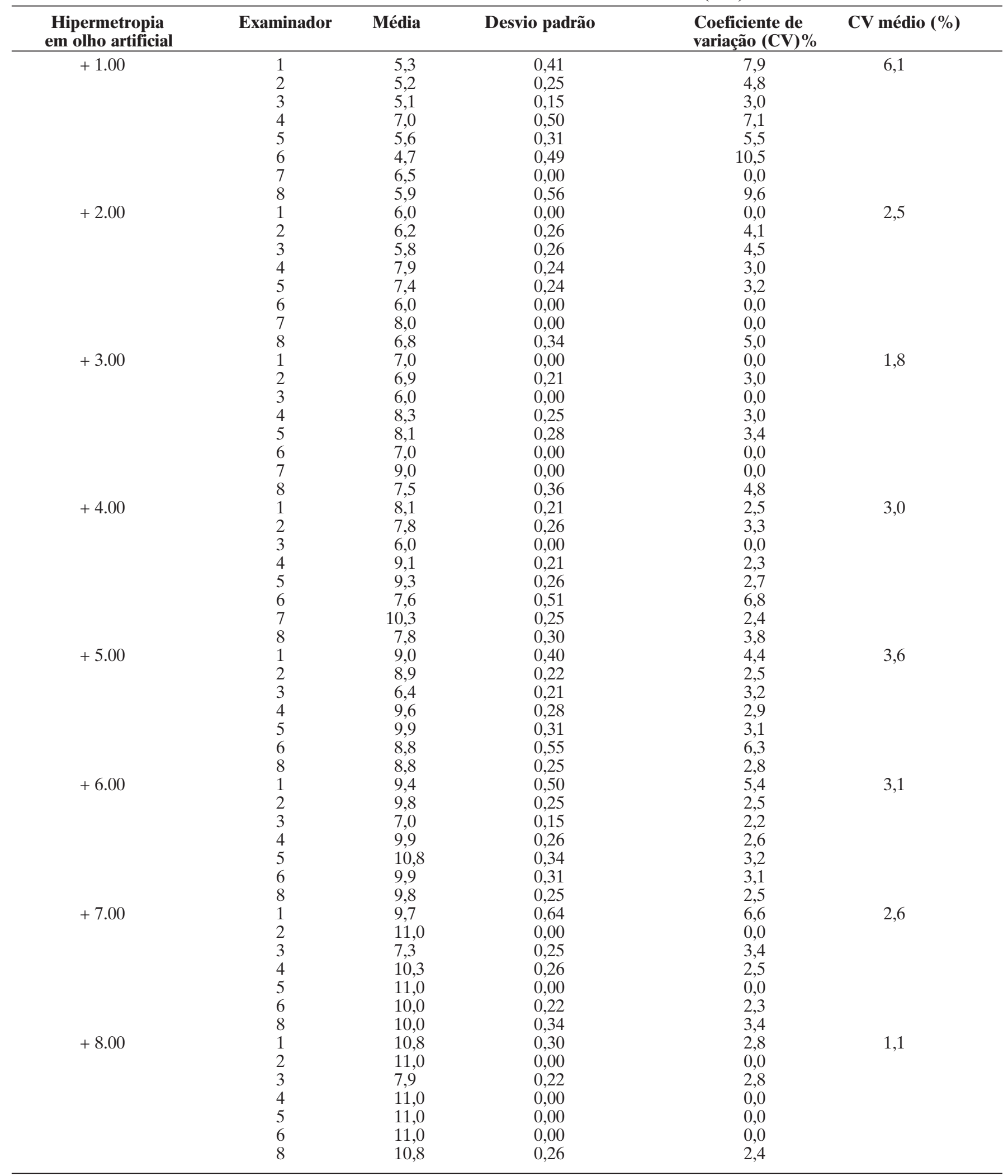

Notas: 1) Sem informação do examinador 7 nas hipermetropias $\geq+5$

2) O desvio-padrão e o coeficiente de variação expressam o erro de mensuração 
Tabela 2

Resultados da retinoscopia sem lentes com leitura em ohms (Ù) no multímetro

\begin{tabular}{|c|c|c|c|c|c|}
\hline $\begin{array}{l}\text { Hipermetropia } \\
\text { em olho artificial }\end{array}$ & Examinador & Média & Desvio padrão & $\begin{array}{r}\text { Coeficiente de } \\
\text { variaç }\end{array}$ & $\begin{array}{l}\text { CV médio (\%) } \\
\text { V) \% }\end{array}$ \\
\hline \multirow[t]{5}{*}{+1.00} & 1 & 1,13 & 0,11 & 9,5 & 8,5 \\
\hline & 3 & 1,14 & 0,10 & 8,3 & \\
\hline & 4 & 1,97 & 0,19 & 9,5 & \\
\hline & 5 & 1,96 & 0,11 & 5,7 & \\
\hline & 8 & 2,26 & 0,04 & 1,8 & \\
\hline \multirow[t]{5}{*}{+2.00} & 1 & 1,33 & 0,09 & 7,0 & 6,1 \\
\hline & 2 & 1,30 & 0,13 & 9,6 & \\
\hline & 3 & 1,31 & 0,06 & 4,7 & \\
\hline & 4 & 2,32 & 0,12 & 5,1 & \\
\hline & 5 & 2,45 & 0,15 & 6,0 & \\
\hline \multirow{7}{*}{+3.00} & 2 & 1,48 & 0,11 & 7,3 & \\
\hline & 3 & 1,46 & 0,03 & 1,9 & \\
\hline & 4 & 2,60 & 0,14 & 5,5 & \\
\hline & 5 & 2,70 & 0,17 & 6,3 & \\
\hline & 6 & 1,34 & 0,06 & 4,7 & \\
\hline & 7 & 2,78 & 0,04 & 1,3 & \\
\hline & 8 & 1,99 & 0,07 & 3,3 & \\
\hline \multirow[t]{6}{*}{+4.00} & 1 & 2,03 & 0,06 & 3,0 & 4,5 \\
\hline & 2 & 1,82 & 0,15 & 8,1 & \\
\hline & 3 & 1,55 & 0,06 & 3,5 & \\
\hline & 4 & 2,81 & 0,10 & 3,6 & \\
\hline & 5 & 2,95 & 0,14 & 4,6 & \\
\hline & 6 & 1,60 & 0,07 & 4,5 & \\
\hline+5.00 & 8 & 1,12 & 0,09 & 7,8 & \\
\hline \multirow[t]{7}{*}{+6.00} & 1 & 2,44 & 0,19 & 7,7 & 7,5 \\
\hline & 2 & 2,59 & 0,08 & 3,0 & \\
\hline & 3 & 1,80 & 0,04 & 2,4 & \\
\hline & 4 & 3,31 & 0,14 & 4,1 & \\
\hline & 5 & 3,35 & 0,22 & 6,5 & \\
\hline & 6 & 2,16 & 0,33 & 15,3 & \\
\hline & 8 & 0,44 & 0,06 & 13,9 & \\
\hline \multirow[t]{7}{*}{+7.00} & 1 & 2,70 & 0,17 & 6,3 & 4,2 \\
\hline & 2 & 2,76 & 0,06 & 2,0 & \\
\hline & 3 & 1,92 & 0,03 & 1,7 & \\
\hline & 4 & 3,26 & 0,17 & 5,3 & \\
\hline & 5 & 3,54 & 0,00 & 0,1 & \\
\hline & 6 & 2,23 & 0,15 & 6,5 & \\
\hline & 8 & 0,42 & 0,03 & 7,4 & \\
\hline \multirow[t]{5}{*}{+8.00} & 1 & 2,86 & 0,06 & 2,1 & 3,7 \\
\hline & 3 & 1,98 & 0,04 & 2,0 & \\
\hline & 4 & 3,56 & 0,00 & 0,0 & \\
\hline & 6 & 2,59 & 0,10 & 3,9 & \\
\hline & 8 & 0,28 & 0,03 & 10,4 & \\
\hline
\end{tabular}

Notas:1) Sem informação dos examinadores 2 e 5 na hipermetropia de +8 e do examinador 7 nas hipermetropias $\geq+5$ 2) O desvio-padrão e o coeficiente de variação expressam o erro de mensuração 
Tabela 3

Confiabilidade (variabilidade do examinador) avaliada pelo coeficiente de correlação intraclasse, segundo os valores de hipermetropia e os modos da retinoscopia sem lente

\begin{tabular}{|c|c|c|c|c|c|c|}
\hline \multirow[t]{3}{*}{ Hipermetropia de } & \multirow{3}{*}{ CCIC } & \multirow{2}{*}{\multicolumn{2}{|c|}{$\begin{array}{c}\text { Leitura em mm } \\
\text { Intervalo de confiança de } 95 \%\end{array}$}} & \multicolumn{3}{|c|}{ Leitura em Ù } \\
\hline & & & & \multirow{2}{*}{ CCI } & \multicolumn{2}{|c|}{ Intervalo de confiança de $\mathbf{9 5} \%$} \\
\hline & & Limite inferior & Limite superior & & Limite inferior & Limite superior \\
\hline+1.00 & 0,805 & 0,631 & 0,946 & 0,968 & 0,927 & 0,992 \\
\hline+2.00 & 0,946 & 0,881 & 0,987 & 0,973 & 0,938 & 0,993 \\
\hline+3.00 & 0,959 & 0,908 & 0,990 & 0,972 & 0,935 & 0,993 \\
\hline+4.00 & 0,956 & 0,901 & 0,989 & 0,984 & 0,962 & 0,996 \\
\hline+5.00 & 0,926 & 0,822 & 0,987 & 0,961 & 0,907 & 0,992 \\
\hline+6.00 & 0,937 & 0,854 & 0,986 & 0,969 & 0,926 & 0,994 \\
\hline+7.00 & 0,939 & 0,860 & 0,987 & 0,989 & 0,972 & 0,998 \\
\hline+8.00 & 0,978 & 0,947 & 0,996 & 0,998 & 0,994 & 1,000 \\
\hline
\end{tabular}

CCIC - coeficiente de correlação intraclasse

Colocavam-se sobre o olho artificial emétrope sucessivamente lentes esféricas de $-1,00 ;-2,00$; etc. a $-8,00 \mathrm{D}$; transformando-o respectivamente em hipermétrope de $+1,00$ a $+8,00$ D.

No olho artificial assim preparado procedia-se à retinoscopia sem utilizar lentes de neutralização.

Para isto movimentava-se o retinoscópio observando no fundo do olho artificial a movimentação correspondente da faixa enquanto se deslocava a luva para cima a partir do ponto zero. No momento da neutralização anotava-se o número do traço da escala milimétrica ou o valor em ohms (Ù ) no mostrador do multímetro. A observação era realizada 20 vezes para cada valor de hipermetropia pelos oito médicos examinadores. Foram feitas 160 observações para cada potência dióptrica entre $+1,00$ e $+4,00 \mathrm{D}$. Para valores entre $+5,00$ e $+8,00 \mathrm{D}$ fizeram-se 120 observações.

Calculou-se a média e o desvio padrão das observações em mm e em ohms (Ù ). Para comparar estas grandezas diferentes calculou-se o coeficiente de variação $(\mathrm{CV})$ a partir dos desvios padrão de cada uma delas. O CV foi considerado indicador da precisão do método por cada examinador, de mensuração.

Como alternativa de análise utilizou-se também o coeficiente de correlação intraclasse (CCIC) com a mesma finalidade.

\section{Resultados}

A tabela 1 mostra os resultados das medidas em milímetros e a tabela 2 os resultados em ohms(Ù ).

O CV médio apresentado nas tabelas 1 e 2 expressa a média dos diversos coeficientes de variação para cada examinador

A tabela 3 mostra a confiabilidade (variabilidade do examinador) medida pelo CCIC. Neste, quanto mais próximo de 1 for o resultado, melhor a precisão.

\section{Discussão}

O estudo permitiu avaliar a acurácia e a precisão das medidas das hipermetropias feitaspelo mesmo médico e por outros médicos, por meio do método de retinoscopia em faixa sem lentes nos valores de $+1,00$ a $+8,00 \mathrm{D}$. Foi mais fácil avaliar hipermetropias entre $+1,00$ e $+4,00 \mathrm{D}$ do que aquelas entre $+5,00 \mathrm{e}+8,00 \mathrm{D}$.

$\mathrm{Na}$ leitura em mm a acurácia e a precisão das medidas foi mais alta nas hipermetropias de $+2,00$ e $+3,00 \mathrm{D}$ com $\mathrm{CV} 0,0 \%$ respectivamente para 3 e 4 examinadores. $\mathrm{Na}$ hipermetropia de $+4,00$ o $\mathrm{CV}$ de $0,0 \%$ foi alcançado por um único examinador. Na leitura em $\dot{U}$ os $\mathrm{CV}$ foram mais altos, o que se pode atribuir à maior sensibilidade do potenciômetro.

Constata-se alguma heterogeneidade entre os examinadores. Na hipermetropia de $+1,00 \mathrm{D}$ : à leitura em mmo CV do examinador $n^{\circ} 6$ foi de $10,5 \%$, enquanto do $\mathrm{n}^{\circ} 7$ foi de $0,0 \%$. Na hipermetropia de $+2,00 \mathrm{D}$ o $\mathrm{CV}$ dos examinadores $\mathrm{n}$ os $1 ; 6$ e 7 ficou em $0,0 \%$; tendo atingido $5,0 \%$ em um só examinador. Comparando todos os resultados percebese ter havido precisão mais alta para as hipermetropias de $+1,00$ a $+5,00$ D.

A acurácia e a precisão se mostraram dependentes de cada examinador. Na média, ambos os modos (leitura em $\mathrm{mm}$ e em Ù) se mostraram bons.

À proporção que a hipermetropia aumenta vai se reduzindo a distância entre os pontos de neutralização dos valores dióptricos, o que dificulta e acaba por impossibilitar sua determinação. A maioria dos examinadores a sentiu a partir de $+6,00 \mathrm{D}$, e um deles já a partir de $+5,00 \mathrm{D}$.

A semelhança entre valores de CV (os quais em princípio indicariam boa reprodutibilidade nas hipermetropias acima de $+5,00 \mathrm{D}$ (Tabelas 1 e 2) parecem representar realmente a dificuldade em distinguir os pontos de neutralização.

As escalas para melhorar a acurácia e a precisão da 
retinoscopia em faixa foram publicadas por Sims ${ }^{(3)}$ e por Wallace et al. ${ }^{(2)}$ que comentam a dificuldade do aprendizado do método, especialmente a determinação da neutralização das hiper-metropias. Não se encontraram publicações com resultados de mais de um examinador.

As análises do desempenho de cada um e entre os oito examinadores permitiu confirmar a possibilidade de reproduzir o método até $+5,00 \mathrm{D}$ tanto no modo de leitura em milímetros quanto naquele com leitura em ohms.

A retinoscopia com leitura em milímetros pareceu discretamente mais precisa que aquela com leitura em ohms. Esperava-se mais precisão na leitura ao potenciômetro do que na escala milimétrica, o que não sucedeu. Presume-se maior dificuldade no aprendizado com aquele, muito mais sensível, do que com esta.

A confiabilidade (variabilidade do examinador) avaliada pelo CCIC foi excelente (Tabela 3). A maioria dos valores ficou acima de 0,9 em ambos os modos (leitura em mm e em Ù), o que mostra margens mínimas de erros entre os examinadores.

Apesar das diferenças observadas os resultados foram homogêneos entre os dois modos, bem como entre os oito examinadores, o que mostra sua reprodutibilidade em olho artificial. Estudos em olhos de pacientes serão necessários para conclusões clínicas.

\section{ReFERÊNCIAS}

1. Corboy JM. The retinoscopy book: an introductory manual for eye care. 5th ed. Thorofare, NJ: Slack; 2003.

2. Wallace DK, Carlin DS, Wright JD. Evaluation of the accuracy of estimation retinoscopy. J AAPOS. 2006;10(3): 232-

3. Sims CN. Retinoscopy assembly with scale. United States Patent no $5650839,1997$. 